\title{
Building and maintaining the epithelium of the lung
}

\author{
Craig R. Rackley ${ }^{1}$ and Barry R. Stripp ${ }^{1,2}$ \\ 1Division of Pulmonary, Allergy and Critical Care, Department of Medicine, and 2Department of Cell Biology, \\ Duke University Medical Center, Durham, North Carolina, USA.
}

\begin{abstract}
Airspaces of the lung are lined by an epithelium whose cellular composition changes along the proximal-to-distal axis to meet local functional needs for mucociliary clearance, hydration, host defense, and gas exchange. Advances in cell isolation, in vitro culture techniques, and genetic manipulation of animal models have increased our understanding of the development and maintenance of the pulmonary epithelium. This review discusses basic cellular mechanisms that regulate establishment of the conducting airway and gas exchange systems as well as the functional maintenance of the epithelium during postnatal life.
\end{abstract}

\begin{abstract}
Introduction
Lung diseases often involve complex changes in lung architecture and function that may only be revealed at the time of clinical presentation. Management is frequently aimed at alleviating symptoms and slowing the course of disease progression. This approach is taken with variable success and without necessarily understanding the principal cause of the pathology. However, as interest in the underlying basis for disease has grown, it has become apparent that the epithelial lining of the lungs is a key disease mediator and target for therapeutic interventions aimed at either preventing disease in susceptible individuals or reversing it in chronically ill patients. Here, we review the development of the pulmonary epithelium, its roles in normal lung function, and the cellular and molecular mechanisms regulating epithelial maintenance. This knowledge is fundamental to understanding the aberrant repair and subsequent remodeling commonly seen in lung disease pathology and to developing therapeutic strategies aimed at treating patients.
\end{abstract}

\section{The functional basis for pulmonary epithelial cell composition and structure}

The branched structure of the human airways allows for efficient bidirectional transfer of approximately six liters of air per minute between the external environment and the alveoli. Harmful contaminants that enter the lung include particulates, pathogenic microorganisms, and gaseous pollutants. Where these agents are deposited and subsequently interact with the epithelium of the lung is dependent upon their physicochemical properties and the flow of air through the airway (1). This, in turn, is influenced by a combination of airway branching, flexibility, and caliber, which dictates velocity and turbulence (2). The higher velocity and turbulent flow of air in proximal airways creates conditions for the impaction of larger particulates and the "scrubbing" of watersoluble contaminants from inspired air. Smaller particulates and less-water-soluble agents can, in contrast, reach deeper into the lung, where they encounter a much larger combined surface area and lower rates of air flow that favor deposition by diffusion. Secretions that line the airway surface trap airborne contaminants, which can then be removed through mucociliary transport; this

Conflict of interest: The authors have declared that no conflict of interest exists. Citation for this article: J Clin Invest. 2012;122(8):2724-2730. doi:10.1172/JCI60519. is a physical clearance mechanism that moves airway secretions and adsorbed contaminants from the closed-ended distal airways toward the oropharynx (3). Mucociliary transport is facilitated by the formation of two separate layers of airway surface liquid: a periciliary layer of less-viscous fluid that allows for the free movement of cilia and an overlying, viscous layer of mucus that serves to transport adsorbed particles and pathogens out of the airways (4).

Gas exchange occurs primarily across the attenuated blood-air barrier that is formed by the juxtaposition of alveolar type I (ATI) epithelial cells and microcapillary endothelial cells. The other specialized alveolar epithelial cell type, the ATII cell, functions in the biosynthesis of pulmonary surfactant, which lowers the surface tension within the alveoli, allowing for respiration at normal transpulmonary pressures. In addition, both cell types play important roles in fluid and ion transport (5).

The pulmonary epithelium fulfills multiple functions essential for tissue homeostasis (6). Different regional needs for functions such as host defense or gas exchange are matched by differences in the type and abundance of specialized epithelial cells at each location. The properties and fate of lung epithelial cells are largely dictated during development, but their phenotype and function are dynamic and can be influenced by changing micro- or macro-environmental conditions. The capacity of the epithelium to adapt to changing environmental conditions is an important property that allows it to tolerate chronic stress. However, exaggerated responses to chronic stimuli or aberrant responses to naive stimuli may result in pathological remodeling, leading to changes in the barrier properties of the epithelium, composition of extracellular lining fluid, and rate of mucociliary transport, all of which have the potential to compromise pulmonary mechanics and gas exchange.

\section{Proximodistal patterning of the developing lung}

The process of lung development in mammals has been best characterized using rodent models. Gastrulation of the developing embryo results in the formation of the three embryonic germ layers whose interactions establish a highly stereotyped body plan. Formation of the anterior foregut pocket results in the establishment of a primitive tube of endoderm surrounded by mesoderm (7). Interactions between these cell types result in the localized formation of primordial buds that give rise to the thymus, lung, liver, and pancreas. The lungs, distal to the trachea, are derived from buds that form from the ventral foregut endoderm at embryonic 


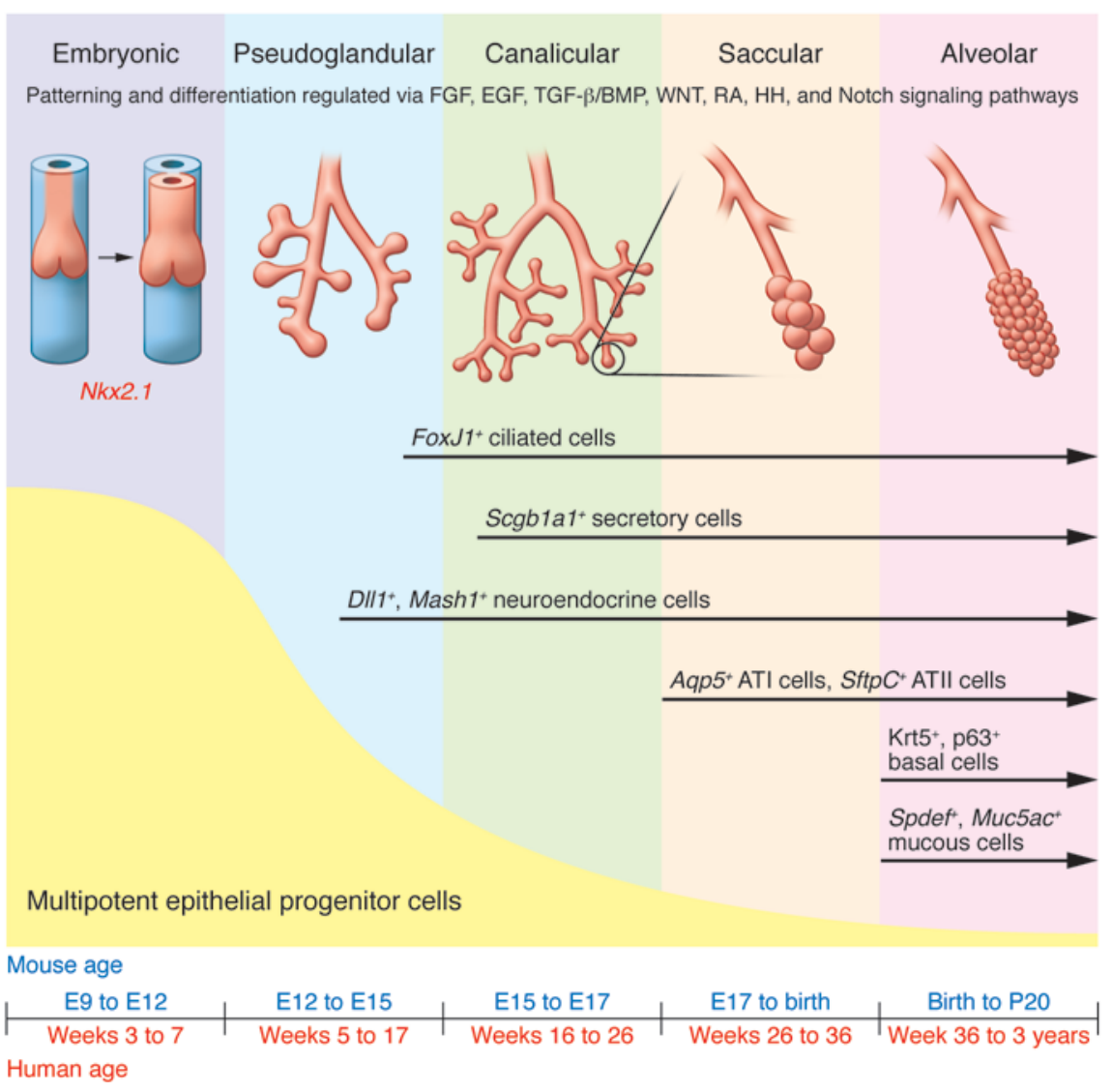

\section{Figure 1}

Lung development in both mouse and human progresses through five overlapping phases based on successive branching: embryonic, pseudoglandular, canalicular, saccular, and alveolar (9). The epithelium is initially composed of multipotent progenitor cells that proliferate and differentiate through development to yield more restricted, differentiated progeny that make up the developed lung epithelium. Signaling pathways that maintain the multipotent progenitor pool are indicated. $\mathrm{HH}$, hedgehog; RA, retinoic acid. day 9.5 in the mouse or week 4 in humans (7-9). The trachea, in contrast, is established by division of the existing foregut lumen into two independent luminal structures that represent the trachea and esophagus $(10,11)$. This early stage of lung development is referred to as the embryonic phase, the first of five phases that were initially defined based upon the changing morphology of developing airways (ref. 9 and Figure 1). The morphologic changes seen in the embryonic phase of lung development are not cell intrinsic, but rather mediated by diffusible signals from surrounding mesenchyme (12). Early endoderm isolated from surrounding mesenchyme loses expression of genes that mark early cell fate decisions, but these markers are completely restored by coculturing endoderm with mesenchyme and partially rescued by the addition of FGF4 (12). Other diffusible signals from the surrounding mesenchyme that have been shown to affect early embryonic cell fate decisions include members of the TGF- $\beta$ /bone morphogenetic protein (TGF- $\beta /$ BMP), Hedgehog, WNT, FGF, and EGF families (12).

As lung development progresses, embryonic endoderm undergoes progressive fate decisions that generate epithelial progenitor cells with increasingly restricted developmental potential over time. The earliest markers of cells that distinguish future lung from other derivatives of the foregut endoderm include expression of the transcription factor $N k \times 2.1$ and reduced expression of the transcription factor Sox2 $(8,10)$. Much like the mesenchymal interactions required by the early endoderm for early differentiation, patterning of the lung during the process of branching morphogenesis is dependent on the local paracrine microenvironments, which differ significantly along the proximodistal axis. Grafting experiments using rat embryos have demonstrated that epithe- lium from both tracheal rudiments and distal lung tips retain the ability to undergo either tracheal or distal lung epithelial cell differentiation under the influence of corresponding regional embryonic mesoderm (13). This demonstrates that early lung endoderm is multipotent and that its fate is dependent upon signals provided by the local microenvironment (primarily mesoderm). However, the capacity of proximal epithelium to undergo multipotent differentiation was lost when early embryonic distal tip mesenchyme was grafted adjacent to proximal epithelium of late pseudoglandular stage lungs (14). The change in epithelial differentiation potential during the transition from pseudoglandular to canalicular phases of lung development reflect the progressive lineage restriction that occurs with advancing gestational age.

Epithelial cells start to assume differentiated characteristics during the pseudoglandular period of lung development. However, the precise temporospatial pattern of this process is highly region and cell type-specific. Some epithelial cell types, such as ciliated cells and neuroendocrine cells, show a proximal-to-distal wave of differentiation that progresses during the phase of rapid airway branching. FoxJ1, a marker of ciliated cell differentiation, first appears in the mouse trachea and large bronchial airways as early as E14 yet its appearance in distal bronchioles is not apparent until E15.5 or later (15). Similarly, pulmonary neuroendocrine cells first appear as precursor cells in proximal conducting airways during the early pseudoglandular period of development and later form at the site of airway branches in more distal conducting airways $(16,17)$. Solitary neuroendocrine precursor cells become innervated by ganglion cells and expand in numbers to generate clusters commonly referred to as neuroepithelial bodies 


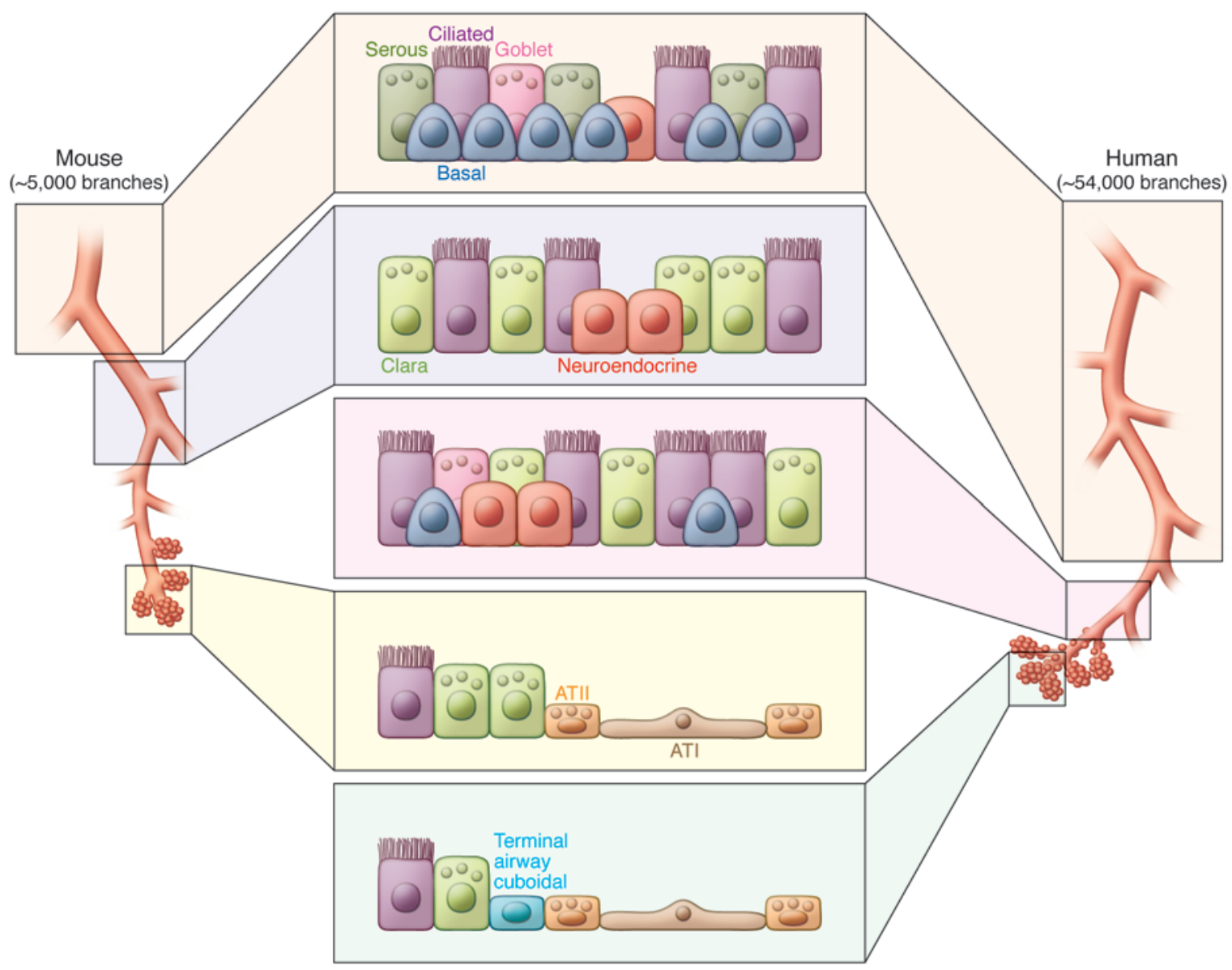

\section{Figure 2}

Regional differences in the cellular composition of the lung epithelium. Within the mouse lung, basal cells are restricted to the tracheobronchial epithelium, which is also populated with secretory, ciliated, and neuroendocrine cells to form a pseudostratified epithelium. Beyond the most proximal bronchi, the airway of the mouse is composed of a simple columnar epithelium that consists of secretory, ciliated, and neuroendocrine cells. In human lungs basal cells are present in diminishing numbers all the way to the level of terminal bronchiole. Respiratory bronchioles are lined by a poorly characterized cuboidal epithelium that leads to the alveolar compartment lined with ATI and ATII cells. Putative progenitor cells have been identified using in vivo lineage-tracing experiments (Clara and basal cells) or proposed based on their capacity to proliferate and differentiate in culture (Clara, basal, and ATII cells). The epithelium of the mouse trachea and proximal bronchi is maintained by airway basal cells (39, 78). Within the bronchiolar region, a population of Clara cell secretory protein-expressing (CCSP-expressing) cells localized the neuroepithelial body in the proximal bronchiole and a population of naphthalene-resistant CCSP-expressing cells from the distal bronchiole of the mouse serve to regenerate their respective regional epithelia following cell-specific injury $(66,67)$. BASCs, at the bronchioalveolar duct junction, have been suggested to serve as progenitors with the capacity to renew both the bronchiolar and alveolar epithelium (68). Finally, it has been long believed that ATII cells serve as the progenitors that maintain the alveolar epithelium (73).

(17). There is some evidence that neuroepithelial bodies play important roles regulating the proliferation of adjacent cells during development and serve as a focal site of secretory cell differentiation in conducting airways $(15,18-20)$.

Epithelial cell types that appear later in development include secretory cells and basal cells of conducting airways, and type 1 and type 2 pneumocytes of the alveolar epithelium. Scgb1a1, an early marker of secretory cell differentiation, is induced within subpopulations of epithelial cells in developing airways during the late pseudoglandular and early canalicular phases of lung development (ref. 21 and Figure 1). However, maturation of secretory cells occurs over a protracted time frame that spans the late embryonic and postnatal periods. Maturation of secretory cells involves the acquisition of the cellular machinery necessary for metabolism of endogenous or xenobiotic compounds and for secretion of various constituents into the airway lining fluid $(20,22,23)$. Basal cells appear in proximal conducting airways during postnatal maturation of the epithelium. Expression of the transcription factor TRP63 defines the developmental progenitor cell pool that yields the basal cell population and is thought to be required for formation of the pseudostratified airway, as p63-null mice lack basal cells and have a columnar ciliated tracheobronchial epithelium (24). Finally, maturation of the epithelium lining respiratory airways, which yields alveolar epithelial type 1 and type 2 pneumocytes, occurs during the late prenatal and early postnatal periods, in what is referred to as the "alveolar" phase of lung development (refs. 9, 25, and Figure 1). During this period, septation of primitive alveoli and continuing expansion of the microcapillary net- 
work provide the delicate alveolar structure needed for efficient gas transfer across the epithelial-endothelial barrier.

The cellular composition of developing airways can be regulated either by signals that control regional (proximal versus distal) cell fates or by local signals that guide the type and proportions of specialized epithelial cell types. The BMP and WNT pathways are two of the major signaling cascades that regulate proximal-todistal patterning of the airway tree (reviewed extensively in refs. 26-28). Inhibition of either pathway in the developing lung endoderm results in loss of distal epithelial structures and cell types (8, 29-31). In contrast, Notch has recently been shown to regulate the balance between ciliated and nonciliated luminal cells in developing airways (32-34). Signaling events involved in the regulation of lung patterning operate primarily during early embryonic and pseudoglandular phases of lung development; however, those involved in regulation of epithelial cell differentiation function during both embryonic and postnatal stages of lung development in addition to normal homeostasis, repair, and remodeling of the epithelium in adult airways.

\section{Epithelial maintenance in the postnatal lung}

Embryonic lung development and postnatal growth mark periods of dynamic tissue expansion involving intercellular signals that govern the abundance and positional identity of many different cell types. In contrast, epithelial cells of the adult lung are largely quiescent and replaced at a very slow rate. Despite numerous studies investigating lung epithelial maintenance and repair, controversy remains regarding the regenerative cell types and regulatory mechanisms involved. Some studies have suggested that multipotent stem cells either exist in the lung or are recruited from a distant source such as the bone marrow to the site of lung tissue damage (35-37). However, evidence derived largely from animal models suggests that regional endogenous progenitor cells are responsible for normal lung epithelial maintenance (Figure 2). The concept that regional progenitor cells maintain the adult lung epithelium is consistent with the process of developmental lineage restriction and functional differences between epithelial cells of different airway regions described above. The following discussion provides a brief overview of what is known of epithelial progenitor cells that contribute to maintenance of the three major epithelial compartments of the postnatal lung.

Pseudostratified epithelium of proximal conducting airways. Airways lined by a pseudostratified epithelium extend from the trachea to the first two intralobar bronchi in mice and to the distal bronchioles of human airways. Both basal and nonciliated luminal cells have been shown to cycle in the steady-state and contribute to repair of the epithelium of rodent airways. However, lineagetracing studies in mice suggest that only basal cells are capable of long-term self-renewal and differentiation into basal, ciliated, and nonciliated cell types in vivo (38-40). Basal cells are an abundant population of relatively undifferentiated cells that contact the basement membrane but not the airway lumen. The expression of characteristic subsets of intermediate filament proteins (keratin 5 [K5], K6, K14, and K16) distinguish basal cells from luminal epithelial cells. However, basal cells show heterogeneity in their expression of these proteins, with subsets distinguished based upon their expression of K5, K14, or K16 (38, 39, 41, 42). Basal cells can be fractionated from rodent and/or human airways by surface staining for either carbohydrate using Griffonia simplicifonia isolectin B4 (43) or various proteins such as aquaporin 3 (44) and nerve growth factor receptor (39), or through use of transgenic reporter or lineage tracing approaches $(39,41)$. Functional analysis of isolated basal cells suggests significant heterogeneity in their capacity for clonal expansion and generation of differentiating progeny in vitro $(39,41,45)$. A number of signaling molecules and pathways regulate the behavior of basal cells including TRP63, SOX2, WNT, and Notch (24, 46-49). Interestingly, Notch signaling promotes basal cell differentiation into luminal progeny (49), suggesting that interactions between basal cells and their luminal counterparts may determine whether daughter cells undergo renewal (generate more basal cells) or differentiation (generate luminal cells). This level of cross-talk between basal and luminal cells of airways is consistent with findings in other systems of regulatory interactions between progenitor cell types of multilayered epithelia. For example, in the mammary gland epithelium, luminal and basal-like myoepithelial cells are maintained as independent, self-renewing lineages in vivo; however, upon segregation of the two populations, myoepithelial cells retain the capacity for selfrenewal and are able to differentiate into luminal cells. Luminal cells, however, lack the capacity for proliferation in the absence of interactions with myoepithelial cells (50). Altered regulation of signaling pathways such as Notch that mediate cell-cell interactions within the lung epithelium could represent critical determinants of epithelial remodeling such as that seen in squamous metaplasia (51), for which basal cells are the likely precursor (52). In addition to their role in squamous metaplasia, a population of K14-expressing basal cells was demonstrated to be a likely precursor for many non-small cell lung cancers (53).

Even though basal cells represent the progenitor cell of pseudostratified airways with the greatest potential for clonal expansion and differentiation, luminal progenitor cells account for the majority of proliferating cells under resting conditions and following moderate injury (54). However, lineage-tracing studies in mice suggest that nonciliated luminal cells defined by their expression of the Clara cell secretory protein SCGB1A1 (commonly known as CCSP, CC10, or uteroglobin) are replaced over time, presumably by basal cell-derived progeny (40). How these studies in rodent airways relate to the behavior of nonciliated luminal progenitor cells of the human airway remains unclear. The abundance of Clara cells is decreased in human bronchial airways while overall epithelial proliferation is increased in patients with epithelial hyperplasia and squamous metaplasia. This may indicate a role for Clara cells in the regulation of proliferation and normal maintenance within the human bronchi (52). Furthermore, even though nonciliated luminal secretory cells of either rodent or human airways share similarities in their responsiveness to cytokine/growth factor-stimulated mucus production $(55,56)$, there are significant species differences in the ultrastructural, biochemical, and molecular properties of these cell types (57).

Mucous cell metaplasia is an important finding in patients with asthma and chronic obstructive pulmonary disease (COPD), where increased mucus production leads to thicker secretions and contributes to the formation at mucous plugs within the airways. Mucous cells are rare in the absence of allergic or environmental exposures and are markedly increased with exposure to allergen, cigarette smoke, or other inhaled toxicants. Findings from both mouse and human studies suggest that mucous cells have multiple potential progenitors: interleukin-13 has been shown to induce ciliated cells to undergo mucous cell differentiation in a mouse model (58), and Clara cells have been shown to differenti- 
ate into mucus-producing cells in response to allergen exposure $(55,59)$. Roles for both ciliated and Clara cells in human mucous cell differentiation are supported by the finding that markers of mucous cell differentiation colocalize with those of either ciliated or Clara cells in tissue from patients with $\operatorname{COPD}(58,60)$. Finally, Notch signaling has been shown to induce basal cell differentiation into both Clara cells and mucous cells, but it is unclear whether mucous cells differentiate directly from basal cells or through a Clara cell intermediary (49).

Simple columnar/cuboidal epithelium of distal conducting airways. Unlike the human lung, intralobar airways of the mouse lung are lined by a simple epithelium that is largely devoid of basal cells (Figure 2). Basal cells of human airways extend in progressively diminishing numbers into bronchioles as small as $1 \mathrm{~mm}$ in diameter $(61,62)$. Even though nonciliated secretory (Clara) cells of terminal conducting airways in the human lung have been shown to proliferate (60), it is not clear whether basal cells of distal airways also contribute significantly to epithelial maintenance at this airway location. It also remains unclear whether there are differences between basal cells of tracheobronchial versus bronchiolar airways. In mouse and rat airways, nonciliated secretory cells function as an abundant pool of renewing progenitor cells $(40,63)$. These progenitor cells are functionally heterogeneous and include subsets that differ in their contribution toward normal maintenance versus repair following chemical (naphthalene) injury $(64,65)$. Naphthalene-resistant epithelial progenitor cells of distal airways, termed variant Clara cells, reside at branch points in association with neuroepithelial bodies and bronchiolar-alveolar duct junctions (66-68). It has been suggested that bronchiolar-alveolar duct junction-associated bronchoalveolar stem cells (BASCs) not only represent a multipotent stem cell, but also constitute a tumorinitiating population, based upon their ability to express marker genes representative of both bronchiolar and alveolar epithelium in vitro $(68,69)$. However, this concept has been challenged by the finding that alveolar cell types contribute to K-ras-induced adenocarcinomas in mice, and in vivo lineage-tracing studies failed to identify distal conducting airway progenitors capable of yielding both bronchiolar and alveolar epithelium during normal maintenance or following injury with naphthalene or hyperoxia $(40,70)$. However, similar lineage-tracing studies performed using a bleomycin injury model did demonstrate alveolar epithelium derived from an Scgb1a1-positive cell (71). Furthermore, a population of p63-positive distal airway basal cells was implicated in regeneration of both airway and alveolar epithelium following lung injury by H1N1 flu infection (72). These discordant observations highlight the need for further studies to identify and characterize progenitor cells that maintain bronchiolar and alveolar epithelium.

Alveolar epithelium. The absence of robust ex vivo organ culture models to study late stages of lung development and postnatal tissue maintenance make alveolarization and alveolar maintenance difficult to study with the same rigor as early embryonic lung development or postnatal airway maintenance. The highly specialized and delicate structure of the alveolar epithelium renders it susceptible to injury by either mechanical forces such as ventila- tion or chemical agents such as elevated oxygen tension or toxic gases. Furthermore, alveolar destruction is a common pathologic feature of many lung diseases including idiopathic pulmonary fibrosis, emphysema, and acute respiratory distress syndrome. Some of the hurdles to progress in defining cell types and molecular mechanisms that regulate alveolar epithelial repair and regeneration include a lack of specific lineage-tracing and/or imaging methods that allow fate mapping of alveolar epithelial cells in vivo. Furthermore, despite the availability of culture models to expand and characterize alveolar epithelium in vitro, the relevance of these models to in vivo tissue maintenance remains controversial. Therefore, even though it has long been accepted that ATII cells function as a progenitor for renewal of injured alveolar epithelium in rodents and humans (73-75), it is unclear whether progenitor cell function is a uniform characteristic of the entire population of ATII cells or whether ATII cells are sufficient for long-term maintenance of the alveolus. Other cell types that may have potential to contribute to maintenance of the alveolar epithelium include ATI cells (76) and rare alveolar epithelial cells that either express the $\alpha 6 \beta 4$ laminin receptor or have been proposed to represent a multipotent BASC $(68,77)$.

\section{Summary and conclusions}

The pulmonary epithelium is an important line of defense against our external environment and is imperative for normal gas exchange. It develops through a complex interplay between endoderm and mesoderm, and continued intercellular signaling is key to lung homeostasis. It is clear that a heterogeneous population of epithelial progenitor cells has the capacity to maintain the conducting airways, as is the case with the alveolar epithelium. However, a large gap remains in our understanding of regional progenitor cell hierarchies and intercellular signaling pathways responsible for maintenance and remodeling of the human lung. The pathological changes seen in lung diseases are commonly associated with changes in both the epithelium and underlying stroma, indicating complex interactions between these cellular compartments. There is a need for improved methods to isolate and study individual populations of regional epithelial progenitor cells from the mouse and human lung. These technical advances will provide tractable models to reveal basic signaling mechanisms that contribute to normal epithelial maintenance and remodeling, as well as screening tools for drug discovery.

\section{Acknowledgments}

The authors would like to acknowledge support from NIH grants T32HL007538, UO1HL111018, R21HL106122, and from a NASA Specialized Center of Research (NSCOR) program grant. The authors thank Brigid Hogan for helpful suggestions regarding the content of this review.

Address correspondence to: Barry R. Stripp, Room 2075 MSRBII, 106 Research Drive, DUMC BOX 103000, Durham, North Carolina 27710, USA. Phone: 919.668.7762; Fax: 919.684.5266; E-mail: barry.stripp@duke.edu.

\footnotetext{
1. Warheit DB. Interspecies comparisons of lung responses to inhaled particles and gases. Crit Rev Toxicol. 1989;20(1):1-29.

2. Schum M, Yeh HC. Theoretical evaluation of aerosol deposition in anatomical models of mammalian lung airways. Bull Math Biol. 1980;42(1):1-15.
}

3. Wolff RK. Effects of airborne pollutants on mucociliary clearance. Environ Health Perspect. 1986; 66:223-237.

4. Randell SH, Boucher RC. Effective mucus clearance is essential for respiratory health. Am J Respir Cell Mol Biol. 2006;35(1):20-28.
5. Dobbs LG, Johnson MD, Vanderbilt J, Allen L, Gonzalez R. The great big alveolar TI cell: evolving concepts and paradigms. Cell Physiol Biochem. 2010;25(1):55-62.

6. Snyder JC, Teisanu RM, Stripp BR. Endogenous lung stem cells and contribution to disease. 
J Pathol. 2009;217(2):254-264.

7. Wells JM, Melton DA. Vertebrate endoderm development. Annu Rev Cell Dev Biol. 1999;15:393-410.

8. Morrisey EE, Hogan BL. Preparing for the first breath: genetic and cellular mechanisms in lung development. Dev Cell. 2010;18(1):8-23.

9. Burri PH. Fetal and postnatal development of the lung. Annu Rev Physiol. 1984;46:617-628.

10. Que J, et al. Multiple dose-dependent roles for Sox 2 in the patterning and differentiation of anterior foregut endoderm. Development. 2007;134(13):2521-2531.

11. Que J, Choi M, Ziel JW, Klingensmith J, Hogan BL. Morphogenesis of the trachea and esophagus: current players and new roles for noggin and Bmps. Differentiation. 2006;74(7):422-437.

12. Wells JM, Melton DA. Early mouse endoderm is patterned by soluble factors from adjacent germ layers. Development. 2000;127(8):1563-1572.

13. Shannon JM, Nielsen LD, Gebb SA, Randell SH. Mesenchyme specifies epithelial differentiation in reciprocal recombinants of embryonic lung and trachea. Dev Dyn. 1998;212(4):482-494.

14. Shannon JM. Induction of alveolar type II cell differentiation in fetal tracheal epithelium by grafted distal lung mesenchyme. Dev Biol. 1994;166(2):600-614.

15. Rawlins EL, Ostrowski LE, Randell SH, Hogan BL. Lung development and repair: contribution of the ciliated lineage. Proc Natl Acad Sci U S A. 2007;104(2):410-417.

16. Sorokin SP, Hoyt RF Jr, Grant MM. Development of neuroepithelial bodies in fetal rabbit lungs. I. Appearance and functional maturation as demonstrated by high-resolution light microscopy and formaldehyde-induced fluorescence. Exp Lung Res. 1982;3(3-4):237-259.

17. Sorokin SP, Hoyt RF Jr, Shaffer MJ. Ontogeny of neuroepithelial bodies: correlations with mitogenesis and innervation. Microsc Res Tech. 1997;37(1):43-61.

18. Hoyt RF Jr, McNelly NA, McDowell EM, Sorokin SP. Neuroepithelial bodies stimulate proliferation of airway epithelium in fetal hamster lung. Am J Physiol. 1991;260(4 pt 1):L234-L240.

19. Hoyt RF Jr, Sorokin SP, McDowell EM, McNelly NA. Neuroepithelial bodies and growth of the airway epithelium in developing hamster lung. Anat Rec. 1993;236(1):15-22.

20. Khoor A, Gray ME, Singh G, Stahlman MT. Ontogeny of Clara cell-specific protein and its mRNA: their association with neuroepithelial bodies in human fetal lung and in bronchopulmonary dysplasia. J Histochem Cytochem. 1996;44(12):1429-1438.

21. Zemke AC, et al. Molecular staging of epithelial maturation using secretory cell-specific genes as markers. Am J Respir Cell Mol Biol. 2009;40(3):340-348.

22. Fanucchi MV, Murphy ME, Buckpitt AR, Philpot RM, Plopper CG. Pulmonary cytochrome P450 monooxygenase and Clara cell differentiation in mice. Am J Respir Cell Mol Biol. 1997;17(3):302-314.

23. Cardoso WV, et al. Secretory product expression during Clara cell differentiation in the rabbit and rat. Am J Physiol. 1993;264(6 pt 1):L543-L552.

24. Daniely Y, et al. Critical role of p63 in the development of a normal esophageal and tracheobronchial epithelium. Am J Physiol Cell Physiol. 2004; 287(1):C171-C181.

25. Amy RW, Bowes D, Burri PH, Haines J, Thurlbeck WM. Postnatal growth of the mouse lung. J Anat. 1977;124(pt 1):131-151.

26. Morrisey EE, Hogan BL. Preparing for the first breath: genetic and cellular mechanisms in lung development. Dev Cell. 2010;18(1):8-23.

27. Warburton D, Schwarz M, Tefft D, Flores-Delgado $\mathrm{G}$, Anderson KD, Cardoso WV. The molecular basis of lung morphogenesis. Mech Dev. 2000;92(1):55-81.

28. Cardoso WV. Molecular regulation of lung development. Annu Rev Physiol. 2001;63:471-494.

29. Mucenski ML, et al. beta-Catenin is required for specification of proximal/distal cell fate during lung morphogenesis. J Biol Chem. 2003; 278(41):40231-40238.

30. Shu W, et al. Wnt/beta-catenin signaling acts upstream of N-myc, BMP4, and FGF signaling to regulate proximal-distal patterning in the lung. Dev Biol. 2005;283(1):226-239.

31. Weaver M, Yingling JM, Dunn NR, Bellusci S, Hogan BL. Bmp signaling regulates proximal-distal differentiation of endoderm in mouse lung development. Development. 1999;126(18):4005-4015.

32. Tsao PN, Vasconcelos M, Izvolsky KI, Qian J, Lu J, Cardoso WV. Notch signaling controls the balance of ciliated and secretory cell fates in developing airways. Development. 2009;136(13):2297-2307.

33. Guseh JS, et al. Notch signaling promotes airway mucous metaplasia and inhibits alveolar development. Development. 2009;136(10):1751-1759.

34. Morimoto M, Liu Z, Cheng HT, Winters N, Bader D, Kopan R. Canonical Notch signaling in the developing lung is required for determination of arterial smooth muscle cells and selection of Clara versus ciliated cell fate. J Cell Sci. 2010;123(pt 2):213-224.

35. Kajstura J, et al. Evidence for human lung stem cells. N Engl J Med. 2011;364(19):1795-1806.

36. Krause DS, et al. Multi-organ, multi-lineage engraftment by a single bone marrow-derived stem cell. Cell. 2001;105(3):369-377.

37. Krause DS. Bone marrow-derived cells and stem cells in lung repair. Proc Am Thorac Soc. 2008;5(3):323-327.

38. Hong KU, Reynolds SD, Watkins S, Fuchs E, Stripp BR. Basal cells are a multipotent progenitor capable of renewing the bronchial epithelium. Am J Pathol. 2004;164(2):577-588.

39. Rock JR, et al. Basal cells as stem cells of the mouse trachea and human airway epithelium. Proc Natl Acad Sci U S A. 2009;106(31):12771-12775.

40. Rawlins EL, et al. The role of Scgb1a1+ Clara cells in the long-term maintenance and repair of lung airway, but not alveolar, epithelium. Cell Stem Cell. 2009;4(6):525-534.

41. Schoch KG, Lori A, Burns KA, Eldred T, Olsen JC, Randell SH. A subset of mouse tracheal epithelial basal cells generates large colonies in vitro. Am J Physiol Lung Cell Mol Physiol. 2004;286(4):L631-L642.

42. Ghosh M, et al. Context-dependent differentiation of multipotential keratin 14-expressing tracheal basal cells. Am J Respir Cell Mol Biol. 2011;45(2):403-410.

43. Randell SH, Comment CE, Ramaekers FC, Nettesheim P. Properties of rat tracheal epithelial cells separated based on expression of cell surface alpha-galactosyl end groups. Am J Respir Cell Mol Biol. 1991;4(6):544-554.

44. Avril-Delplanque A, Casal I, Castillon N, Hinnrasky J, Puchelle E, Peault B. Aquaporin-3 expression in human fetal airway epithelial progenitor cells. Stem Cells. 2005;23(7):992-1001.

45. Ghosh M, et al. A single cell functions as a tissuespecific stem cell and the in vitro niche-forming cell. Am J Respir Cell Mol Biol. 2011;45(3):459-469.

46. Que J, Luo X, Schwartz RJ, Hogan BL. Multiple roles for Sox 2 in the developing and adult mouse trachea. Development. 2009;136(11):1899-1907.

47. Brechbuhl HM, et al. beta-catenin dosage is a critical determinant of tracheal basal cell fate determination. Am J Pathol. 2011;179(1):367-379.

48. Giangreco $A$, et al. $\beta$-Catenin determines upper airway progenitor cell fate and preinvasive squamous lung cancer progression by modulating epithelial-mesenchymal transition. J Pathol. 2012;226(4):575-587.

49. Rock JR, Gao X, Xue Y, Randell SH, Kong YY, Hogan BL. Notch-dependent differentiation of adult airway basal stem cells. Cell Stem Cell. 2011; 8(6):639-648.

50. Van Keymeulen A, et al. Distinct stem cells contribute to mammary gland development and maintenance. Nature. 2011;479(7372):189-193.

51. Voynow JA, Fischer BM, Roberts BC, Proia AD. Basal-like cells constitute the proliferating cell popula- tion in cystic fibrosis airways. Am J Respir Crit Care Med. 2005;172(8):1013-1018.

52. Barth PJ, Koch S, Muller B, Unterstab F, von Wichert P, Moll R. Proliferation and number of Clara cell $10-\mathrm{kDa}$ protein (CC10)-reactive epithelial cells and basal cells in normal, hyperplastic and metaplastic bronchial mucosa. Virchows Arch. 2000;437(6):648-655.

53. Ooi AT, et al. Presence of a putative tumor-initiating progenitor cell population predicts poor prognosis in smokers with non-small cell lung cancer. Cancer Res. 2010;70(16):6639-6648.

54. Evans MJ, Shami SG, Cabral-Anderson LJ, Dekker NP. Role of nonciliated cells in renewal of the bronchial epithelium of rats exposed to NO2. AmJ Pathol. 1986;123(1):126-133.

55. Evans CM, et al. Mucin is produced by clara cells in the proximal airways of antigen-challenged mice. Am J Respir Cell Mol Biol. 2004;31(4):382-394.

56. Takeyama K, Fahy JV, Nadel JA. Relationship of epidermal growth factor receptors to goblet cell production in human bronchi. Am J Respir Crit Care Med. 2001;163(2):511-516.

57. Plopper CG, Hill LH, Mariassy AT. Ultrastructure of the nonciliated bronchiolar epithelial (Clara) cell of mammalian lung. III. A study of man with comparison of 15 mammalian species. Exp Lung Res. 1980;1(2):171-180.

58. Tyner JW, et al. Blocking airway mucous cell metaplasia by inhibiting EGFR antiapoptosis and IL-13 transdifferentiation signals. J Clin Invest. 2006; 116(2):309-321.

59. Chen $\mathrm{G}$, et al. SPDEF is required for mouse pulmonary goblet cell differentiation and regulates a network of genes associated with mucus production. J Clin Invest. 2009;119(10):2914-2924.

60. Boers JE, Ambergen AW, Thunnissen FB. Number and proliferation of clara cells in normal human airway epithelium. Am J Respir Crit Care Med. 1999; 159(5 pt 1):1585-1591.

61. Evans MJ, Van Winkle LS, Fanucchi MV, Plopper CG. Cellular and molecular characteristics of basal cells in airway epithelium. Exp Lung Res. 2001; 27(5):401-415.

62. Rock JR, Randell SH, Hogan BL. Airway basal stem cells: a perspective on their roles in epithelial homeostasis and remodeling. Dis Model Mech. 2010; 3(9-10):545-556.

63. Evans MJ, Cabral-Anderson LJ, Freeman G. Role of the Clara cell in renewal of the bronchiolar epithelium. Lab Invest. 1978;38(6):648-653.

64. Giangreco A, Arwert EN, Rosewell IR, Snyder J, Watt FM, Stripp BR. Stem cells are dispensable for lung homeostasis but restore airways after injury. Proc Natl Acad Sci U S A. 2009;106(23):9286-9291.

65. Teisanu RM, et al. Functional analysis of two distinct bronchiolar progenitors during lung injury and repair. Am J Respir Cell Mol Biol. 2011;44(6):794-803.

66. Hong KU, Reynolds SD, Giangreco A, Hurley CM, Stripp BR. Clara cell secretory protein-expressing cells of the airway neuroepithelial body microenvironment include a label-retaining subset and are critical for epithelial renewal after progenitor cell depletion. Am J Respir Cell Mol Biol. 2001;24(6):671-681.

67. Giangreco A, Reynolds SD, Stripp BR. Terminal bronchioles harbor a unique airway stem cell population that localizes to the bronchoalveolar duct junction. Am J Pathol. 2002;161(1):173-182.

68. Kim CF, et al. Identification of bronchioalveolar stem cells in normal lung and lung cancer. Cell. 2005;121(6):823-835.

69. Jackson EL, et al. Analysis of lung tumor initiation and progression using conditional expression of oncogenic K-ras. Genes Dev. 2001;15(24):3243-3248.

70. Xu X, et al. Evidence for type II cells as cells of origin of K-Ras-induced distal lung adenocarcinoma. Proc Natl Acad Sci U S A. 2012;109(13):4910-4915.

71. Tropea KA, et al. Bronchioalveolar stem cells increase after mesenchymal stromal cell treat- 
ment in a mouse model of bronchopulmonary dysplasia. Am J Physiol Lung Cell Mol Physiol. 2012; 302(9):L829-L837.

72. Kumar PA, et al. Distal airway stem cells yield alveoli in vitro and during lung regeneration following H1N1 influenza infection. Cell. 2011;147(3):525-538.

73. Adamson IY, Bowden DH. The type 2 cell as progenitor of alveolar epithelial regeneration. A cytodynamic study in mice after exposure to oxygen. Lab Invest. 1974;30(1):35-42.
74. Bachofen M, Weibel ER. Basic pattern of tissue repair in human lungs following unspecific injury. Chest. 1974;65(suppl):14S-19S.

75. Evans MJ, Cabral LJ, Stephens RJ, Freeman G. Transformation of alveolar type 2 cells to type 1 cells following exposure to NO2. Exp Mol Pathol. 1975;22(1):142-150.

76. Gonzalez RF, Allen L, Dobbs LG. Rat alveolar type I cells proliferate, express OCT-4, and exhibit phenotypic plasticity in vitro. Am J Physiol Lung Cell Mol
Physiol. 2009;297(6):L1045-L1055.

77. Chapman HA, et al. Integrin alpha6beta4 identifies an adult distal lung epithelial population with regenerative potential in mice. J Clin Invest. 2011; 121(7):2855-2862.

78. Hong KU, Reynolds SD, Watkins S, Fuchs E, Stripp BR. In vivo differentiation potential of tracheal basal cells: evidence for multipotent and unipotent subpopulations. Am J Physiol Lung Cell Mol Physiol. 2004;286(4):L643-L649. 